\title{
SIGNIFICAÇÕES RELACIONADAS À CIRURGIA BARIÁTRICA: ESTUDO NO PRÉ E PÓS-OPERATÓRIO
}

MEANINGS RELATED TO BARIATRIC SURGERY: A STUDY ON PRE AND POSTOPERATIVE

Recibido: 19 de Mayo del 2015 | Aceptado: 14 de Julio del 2015

\author{
CÍNTIA SILVA 1; ANDRÉ FARO \\ (UNIVERSIDADE FEDERAL DE SERGIPE, São Cristóvão, Sergipe, Brasil)
}

\begin{abstract}
RESUMO
OBJETIVO: conhecer as significações atreladas à cirurgia bariátrica segundo candidatos ao procedimento (que estão na fila de espera e que passaram pelas avaliações pré-operatórias) e indivíduos que realizaram essa cirurgia há, pelo menos, um ano e agora avaliam suas repercussões. METOD0: Participaram 10 pessoas no pré-operatório e 10 pessoas no pós-operatório, ambos os sexos, residentes da cidade de Aracaju/SE. Empregou-se um roteiro de entrevista aberto, orientado pelos eixos temáticos: cirurgia bariátrica, motivações e expectativas. Para análise dos dados utilizou-se o programa IRAMUTEQ. RESULTADOS: A partir de um único corpus, obtiveram-se três classes: Motivações sociais para a realização da cirurgia; Questões clínicas da cirurgia; e Mudanças na alimentação, associadas à cirurgia. Realizou-se, ainda, a análise de similitude da palavra 'psicólogo'. Ao final, viu-se que a cirurgia evoca diferentes sentidos: homens se preocupam mais com questões da saúde e comorbidades aliadas a obesidade, e as mulheres sentem-se pressionadas para ter um corpo perfeito e inquietam-se com a imagem social atrelada ao ser obeso. CONCLUSOES: 0 grupo pré-operatório julga valer à pena o procedimento para se livrar do sofrimento social e físico que 0 peso carrega, já o grupo pós-operatório não fala de preconceito e dificuldades sociais, sugerindo uma melhoria nesses quesitos.
\end{abstract}

PALAVRAS-CHAVES: Cirurgia bariátrica, Pré-operatório, Pós-operatório.

\section{ABSTRACT}

OBJECTIVE: To understand the meanings associated to bariatric surgery. METHOD: Two types of informants participated in this study: one group was composed of candidates on the waiting list for this procedure (they have already done preoperative evaluations); the other group was composed of individuals who underwent surgery a year ago and can now evaluate the impact of it. A total of 20 male and female subjects participated: 10 before surgery, 10 after surgery; all residents of the city of Aracaju/SE. An open interview that included themes such as bariatric surgery and motivations and expectations was applied. Data analysis was done with the IRAMUTEQ program. RESULTS: Three topics emerged: social motivations for the surgery, clinical issues of surgery, and changes in diet linked to surgery. Similarly, an analysis of the word 'psychologist' was done. In the end, surgery evokes different meanings: men are more concerned with health issues and comorbidities related to obesity; women feel pressured to have a perfect body and worry about the social image linked to obesity. CONCLUSION: Subjects before surgery believe the procedure is a way to get rid of physical and social suffering caused by overweight. Prejudice and social difficulties is not a topic discussed by the after surgery subjects, suggesting an improvement in their social wellbeing.

KEY WORDS: Bariatric surgery, Preoperative, Postoperative.

1. Universidade Federal de Sergipe, Departamento de Psicologia, São Cristóvão, Sergipe, Brasil. Contato: Rua Senhor do Bomfim, nº 19, Centro, Macaúbas (Bahia, Brasil). CEP 46500-000. Telefone: +55 61 98781607. Email: cafscinth@hotmail.com

2. Univer sidade Federal de Sergipe, Programa de Pós-Graduação em Psicologia Social, Mestrado em Psicologia Social, São Cristóvão, Sergipe, Brasil. 
No Brasil, de acordo com o censo 20082009, do Ministério da Saúde, 34\% da população de 20 a 59 anos estava com sobrepeso (IMC $\geq 25<30 \mathrm{~kg} / \mathrm{m}^{2}$ ) e $14,5 \%$ da população era considerada obesa (IMC $\geq 30 \mathrm{~kg} / \mathrm{m}^{2}$ ) (Brasil, 2011). Dados da Organização Mundial da Saúde (OMS), veiculados por meio da Sociedade Brasileira de Cirurgia Bariátrica e Metabólica (SBCBM), estimam que em 2015 existam 2,3 bilhões de pessoas com excesso de peso e 700 milhões de obesos no mundo inteiro (http://www.sbcb.org.br).

A cirurgia bariátrica e metabólica, realizada no aparelho digestivo para diminuir a ingestão e absorção de alimentos, é a intervenção mais invasiva para o tratamento da obesidade, utilizada quando os tratamentos dietético, farmacológico e a atividade física não surtem mais resultados, e quando o risco da cirurgia é menor diante da gravidade do quadro de saúde global do indivíduo. 0 conceito metabólico adicionado ao nome foi incorporado pela importância que a cirurgia adquiriu no tratamento de doenças causadas, agravadas ou cujo controle é dificultado pelo excesso de peso - como 0 diabetes e a hipertensão - comorbidades comuns na obesidade (Moliner \& Rabuske, 2008).

Muitos são os fatores que precisam estar adequados para a realização dessa intervenção. Conforme os preceitos médicos, (Brasil, 2013), a indicação cirúrgica é baseada em três critérios: Índice de Massa Corpórea (IMC) acima de 30 $\mathrm{Kg} / \mathrm{m}^{2}$, com a presença de comorbidades, tais como alto risco cardiovascular, diabetes mellitus e/ou hipertensão arterial sistêmica de difícil controle, e 16 anos a idade mínima para realização da cirurgia. 0 critério 'tempo de doença', que diz respeito a duração de tentativas com procedimentos convencionais, deve ser de pelo menos dois anos de insucesso ou recidiva do peso, verificados por meio de dados colhidos do histórico clínico do paciente (Brasil, 2013).
A cirurgia bariátrica requer uma equipe de profissionais da área da saúde que avaliará o paciente, verificando se está apto ou não para esse procedimento tão invasivo. A psicologia tem um papel fundamental nesse processo, inserido como profissional exigido na equipe mínima para avaliar se o paciente está ou não preparado para enfrentar as transformações advindas da cirurgia (Brasil, 2013; Sebastiani \& Maia, 2005). A justificativa para tal é que as mudanças não são somente alimentares, mas também comportamentais. Por trás de um prato de comida, associam-se vários outros sentimentos e crenças que preenchem a vida das pessoas, ganhando, assim, o significado não somente nutritivo do corpo (Machado, Zilberstein, Cecconello, \& Monteiro, 2008).

A atuação do psicólogo no processo cirúrgico pode ocorrer em três momentos distintos, cada um com suas peculiaridades: antes, durante e após a cirurgia, essa última dividida em pós-operatório imediato e tardio (Gabarra \& Crepaldi, 2009). Os psicólogos têm desenvolvido e implantado programas que visam 0 aumento da frequência de comportamentos saudáveis, buscando compreender 0 papel das variáveis psicológicas sobre a manutenção da saúde, o desenvolvimento de doenças e seus comportamentos associados (Almeida \& Malagris, 2011).

A efetivação de um bom acompanhamento psicológico no préoperatório tem influência direta nas reações do paciente durante e no pós-operatório, uma vez que existem relações entre 0 estado emocional do paciente nas três fases citadas da cirurgia (Sebastiani \& Maia, 2005). O objetivo do psicólogo da saúde é contribuir para a melhoria do bem-estar dos indivíduos, incluindo no seu projeto de vida conjuntos de atitudes e comportamentos ativos que promovam a saúde e previnam as doenças, aperfeiçoando também técnicas de enfretamento no processo de ajustamento ao adoecer, à doença e às 
suas eventuais consequências (Almeida \& Malagris, 2011).

Marcando tal importância, sabe-se que pacientes que se submetem à cirurgia sem um prévio acompanhamento psicológico têm um risco maior de desenvolver expectativas irreais quanto ao alcance da cirurgia (Almeida et al., 2011). O resultado comum é a frustração consigo mesmas (Magdaleno, Chaim, \& Turato, 2009), sendo que tensão e ansiedade no pré-operatório estão associadas ao maior risco de depressão, baixa aderência ao programa de reabilitação e outras intercorrências no pósoperatório (Sebastiani \& Maia, 2005).

O obeso pós-operado necessitará de um acompanhamento para modificar sua autoimagem transformada, a fim de perceber-se como novo corpo, mas que não é garantia de realização de seus desejos, muitas vezes idealizados (Marchiolli, Marchiolli, \& Silva, 2005). Geralmente, a estabilização do peso ocorre após 12 meses do procedimento cirúrgico (Magdaleno et al., 2009), sendo um período de especial importância para se averiguar as relações entre 0 que 0 paciente esperava como resultado e 0 que ele observa como repercussões efetivas da cirurgia em seu organismo e no cotidiano.

Após a cirurgia de obesidade, a pessoa tende a sentir satisfação pela perda de peso, mas pode ter dificuldades para reconhecer-se e identificar-se com o corpo magro, sentindo estranheza ou mantendo a imagem corporal anterior sobreposta em seu corpo atual (Moliner \& Rabuske, 2008). Até o momento da cirurgia, por mais que tenham sido orientados e esclarecidos quanto às dificuldades, estavam, por vezes, fascinados com a idéia de resolverem todos os seus problemas a partir da cirurgia, como em um "passe de mágica" (Magdaleno et al., 2009, p. 76).

De acordo com Sociedade Brasileira de Cirurgia Bariátrica e Metabólica (SBCBM) (http://www.sbcb.org.br) foram realizadas, em 2010, 60.000 cirurgias, e esse número vem aumentando nos últimos anos. Por outro lado, ainda que a avaliação psicológica faça parte dos protocolos do procedimento, são poucos os estudos envolvendo cirurgia bariátrica no que diz respeito à psicologia. Na Scielo, em 16 de agosto de 2013, encontrou-se 127 referências com a palavra-chave 'cirurgia bariátrica'. Entretanto este número diz respeito em sua maioria de artigos metodológicos e/ou clínicos da clínicacirúrgica do procedimento. Além disso, um maior número de estudos foi realizado após a cirurgia, sendo que apenas alguns têm tentado compreender características obesas antes da cirurgia bariátrica, ou mesmo fazendo relações entre 0 pré e pósoperatório.

Em contrapartida à importância do acompanhamento psicoterápico, Segal e Fandiño (2002) citam 0 abandono de critérios psicológicos na seleção de candidatos a estes procedimentos, provavelmente devido ausência de instrumentos que permitam adequada acurácia prognóstica, mostrando um julgamento clínico baseado em evidências, cada vez menos subjetivo. A preocupação destes autores é muito pertinente, posto que o procedimento cirúrgico tem sido indicado e realizado com uma frequência cada vez maior, ainda que se constate 0 abandono desses critérios na seleção de candidatos a este procedimento, 0 que interfere no prognóstico dos pacientes operados (Marcelino \& Patrício, 2011). Isso foi pontuado também na realidade de outros países, tal qual destacaram Van Hout, Boekestein, Fortuin, Pelle e Van Heck (2006a), que chamaram a atenção para as falhas da cirurgia bariátrica, frequentemente atribuídas a fatores psicológicos e/ou transtornos alimentares, ao invés de fatores técnicos.

De todo o exposto, julga-se importante 0 estudo das razões que a cirurgia tem ou 
teve na vida dos obesos e ex-obesos, as significações ou percepções acerca da cirurgia bariátrica, compreendendo as crenças, expectativas e conhecimento sobre a obesidade e 0 procedimento (Silva \& Maia, 2013). Menciona-se isso pois o uso de indicadores unicamente objetivos, tais como a redução ou não do peso, ou ainda apenas 0 atendimento aos protocolos clínicos, produz resultados alheios aos sentimentos e experiências dos pacientes bariátricos (Engström, Wiklund, Ólsen, Lönroth, \& Forsberg, 2011).

Considerando que as implicações psicológicas são importantes para se compreender a organização e funcionamento psicológico e social do obeso ou do ex-obeso, este estudo teve como objetivo conhecer as significações atreladas à cirurgia bariátrica segundo candidatos ao procedimento (que estão na fila de espera e que passaram pelas avaliações pré-operatórias) e indivíduos que realizaram essa cirurgia há, pelo menos, um ano e agora avaliam suas repercussões.

\section{MÉTODO}

\section{Participantes}

Os participantes eram residentes da cidade de Aracaju (SE) e foram divididos em grupos pré-operatório e pós-operatório. 0 grupo pré-operatório foi composto por oito mulheres e dois homens, com IMC médio de 48,7 [Desvio-Padrão (DP) $=6,88$ ], sendo - IMC mínimo $37,2 \mathrm{Kg} / \mathrm{m}^{2}$ e o máximo 61,5 $\mathrm{Kg} / \mathrm{m}^{2}$. A idade variou entre 17 e 56 anos, média de 37,4 (DP = 12,56). Dos participantes seis eram solteiros e quatro casados; cinco farão a cirurgia pelo Sistema Único de Saúde (SUS) e cinco por meio de planos de saúde privados.

O grupo pós-operatório foi formado por sete mulheres e três homens com idade média de 34 anos ( $D P=11,77$ ), variando de 22 a 50 anos. Quanto ao estado civil, seis eram solteiros e quatro casados. Todos fizeram a cirurgia por convênio e não foram sido submetidas a outros tipos de procedimento, que não a bariátrica ou cirurgias estéticas pós-bariátrica. $O$ IMC antes da cirurgia variava de $38,9 \mathrm{~kg} / \mathrm{m}^{2}$ a 48 $\mathrm{kg} / \mathrm{m}^{2}$, sendo a média $41,6 \mathrm{~kg} / \mathrm{m}^{2}(\mathrm{DP}=3,2)$. O IMC atual, depois da cirurgia, varia entre $22,6 \mathrm{~kg} / \mathrm{m}^{2}$ a $31,1 \mathrm{~kg} / \mathrm{m}^{2}$, com média de 26,9 $\mathrm{kg} / \mathrm{m}^{2}$ (DP $=2,93$ ). Como parâmetro de seleção de candidatos nesse grupo, delimitou-se o tempo mínimo de realização da cirurgia em um ano. $O$ tempo médio de realização da cirurgia dos participantes foi de 3,2 anos (DP $=1,68)$.

\section{Instrumentos e Procedimentos}

Empregou-se um roteiro de entrevista aberto, orientado por eixos temáticos, para coleta dos dados. Os participantes foram contatados por indicação (critério amostral da intencionalidade) e o local da entrevista foi escolhido e acordado juntamente com a pesquisadora. Também foram visitados grupos de preparação para bariátrica, frequentados tanto por pessoas que irão fazer a cirurgia, como também pessoas que já realizaram o procedimento. Utilizou-se um gravador para registro das falas dos participantes e entregou-se o termo de consentimento livre e esclarecido, para que os participantes, depois de informados sobre os objetivos do estudo, declarassem sua aceitação em participar da pesquisa.

Inicialmente coletaram-se dados de caracterização dos participantes, tais como: idade, sexo, tempo de cirurgia (para os que já fizeram), estado civil e IMC (antes e depois no grupo pós-operatório). Posteriormente foi apresentado um roteiro de entrevista aberto com seis eixos temáticos: 1. Motivos/motivações para se fazer a cirurgia; 2 . Expectativas no pósoperatório; 3. Mudanças observadas após a cirurgia; 4. A preparação no pré-cirúrgico; 5. Vida no pós-operatório; 6 . Importância do psicólogo no processo. A cada indivíduo foi solicitado que falassem, de modo livre, acerca do eixo proposto, sendo feitas 
questões de esclarecimento a partir dos discursos produzidos.

\section{Análise de Dados}

Para análise dos dados textuais obtidos com as entrevistas, utilizou-se o programa Interface de $R$ pour les Analyses Multidimensionnelles de Textes et de Questionnaires (IRAMUTEQ) (ver Camargo \& Justo, 2013). Como parâmetro de análise, avaliou-se a Classificação Hierárquica Descendente (CHD), que é uma técnica que possibilita classificar por semelhança os elementos que compõem cada segmento de texto e dividi-los em classes, que são organizadas em um dendograma. Com a CHD, obteve-se o conjunto de termos que compuseram as classes, organizados de forma descendente pelo valor do QuiQuadrado $\left(X^{2}\right)$, sendo que quanto mais alto - Qui-Quadrado, maior 0 nível de associação entre a palavra e a classe. 0 percentual de presença na classe permite uma representação do peso da forma/palavras em cada classe. Para cada classe, atribuiu-se uma nomenclatura derivada da análise dinâmica do conteúdo lexical agrupado na $\mathrm{CHD}$, representando 0 sentido que poderia ser extraído a partir da classe.

Analisou-se também a conexidade entre palavras relevantes através da análise de similitude, baseada na teoria dos grafos, com o objetivo de estudar a proximidade e a relação entre elementos de um conjunto de formas, identificando coocorrências entre as palavras relevantes ao estudo, com os resultados mostrados em um gráfico simples em forma de árvore.

Neste estudo trabalhou-se com um único corpus, que significa o conjunto de dados que se pretende analisar, 0 qual foi composto pela transcrição de todos os eixos das entrevistas. Por meio da associação direta de textos do banco de dados, foi possível fazer uma comparação entre grupos em função das variáveis de caracterização, análise de contraste, que divide o corpus de acordo uma variável escolhida. A principal variável utilizada foi "grupo" (pré-operatório ou pós-operatório). Além dessa, comparou-se também por sexo (masculino ou feminino), idade (até 30 , de 31 à $40,>40$ ), cirurgia (pelo SUS ou por planos de saúde privados), tempo de cirurgia (0, de 1 a 2 anos, de 2 a 3 anos, de 3 a 4 anos, e $>4$ anos) e estado civil (solteiro ou casado).

\section{RESULTADOS E DISCUSSÃO}

A Figura 1 demonstra a divisão das classes construídas por meio da Classificação Hierárquica Descendente (CHD). Para representação do dendograma neste relato, devido ao volume de termos em análise, escolheram-se apenas as formas que exibiram significância em $p<0,0001$. 0 IRAMUTEQ repartiu o corpus em 633 segmentos de texto, três classes, e, em média, 35 palavras por segmento. 0 corpus foi dividido duas vezes, originando as classes 1 e 3 na primeira divisão e a classe 2 na segunda divisão. As referidas classes obtidas através da análise foram nomeadas da seguinte maneira: classe 1, Preocupação com a Imagem Social $(43,9 \%$ dos segmentos de texto); classe 2, Aspectos Clínicos Associados à Cirurgia $(29,2 \%)$ e classe 3, Mudanças na Alimentação $(26,9 \%)$. Dado o exposto, a Figura 1 descreve a temática dos discursos com os conteúdos derivados da análise das classes. 
FIGURA 1.

Dendograma das significações atreladas à cirurgia bariátrica.

\begin{tabular}{|c|c|c|c|c|c|c|c|c|}
\hline & & & \multicolumn{3}{|c|}{ Discursos sobre a cirurgia bariátrica } & & & \\
\hline \multirow{2}{*}{\multicolumn{3}{|c|}{$\begin{array}{l}\text { Classe } 1(43,9 \%) \\
\text { Motivações sociais para a } \\
\text { realização da cirurgia }\end{array}$}} & \multirow{2}{*}{\multicolumn{3}{|c|}{$\begin{array}{c}\text { Classe } 3(26,9 \%) \\
\begin{array}{c}\text { Mudanças na alimentação } \\
\text { associadas à cirurgia }\end{array}\end{array}$}} & & & \\
\hline & & & & & & \multicolumn{3}{|c|}{$\frac{\text { Classe 2 }(29,2 \%)}{\text { Questões clínicas da cirurgia }}$} \\
\hline Palavra & $\mathbf{F}(\%)$ & $\mathrm{X}^{2}$ & Palavra & $\mathbf{F}(\%)$ & $x^{2}$ & Palavra & $\mathbf{F}(\%)$ & $\mathrm{x}^{2}$ \\
\hline Pessoa & 83,91 & 67,55 & Comer & 70,21 & 109,15 & Cirurgia & 58,70 & 47,02 \\
\hline Magro & 86,49 & 29,24 & Doce & 100,0 & 41,97 & Mês & 93,75 & 33,31 \\
\hline Gordo & 69,44 & 22,02 & Dumping & 100,0 & 30,54 & Saúde & 76,92 & 30,19 \\
\hline Mudar & 75,56 & 19,96 & Comida & 63,64 & 24,17 & Médico & 84,21 & 28,90 \\
\hline Preconceito & 100,0 & 19,70 & Passar & 50,00 & 21,19 & Dieta & 80,95 & 28,39 \\
\hline Importância & 100,0 & 18,35 & Beber & 100,0 & 19,53 & Perder & 78,26 & 28,05 \\
\hline \multirow[t]{2}{*}{ Importante } & 80,65 & 18,01 & Só & 50,00 & 18,38 & Expectativa & 75,00 & 25,57 \\
\hline & & & Copo & 100,0 & 16,50 & Líquido & 100,0 & 22,24 \\
\hline \multicolumn{3}{|c|}{ Análise de Contraste } & Lembrar & 87,50 & 15,18 & Já & 48,04 & 21,81 \\
\hline \multirow{2}{*}{\multicolumn{3}{|c|}{$\begin{array}{cc}\text { Grupo pré-operatório } & p=0,00029 \\
\text { Sexo Feminino } & p<0,0001 \\
\end{array}$}} & Carne & 87,50 & 15,18 & Peso & 64,52 & 19,92 \\
\hline & & & & & & Exame & 100,0 & 19,73 \\
\hline & & & \multicolumn{3}{|c|}{ Análise de Contraste } & Começar & 65,52 & 19,63 \\
\hline & & & \multicolumn{3}{|c|}{ Grupo pós-operatório $p=0,00259$} & Minha & 48,75 & 17,50 \\
\hline & & & & & & Tentar & 83,33 & 17,44 \\
\hline & & & & & & Motivo & 66,67 & 17,11 \\
\hline & & & & & & Plano & 88,89 & 15,81 \\
\hline & & & & & & \multicolumn{3}{|c|}{ Análise de Contraste } \\
\hline & & & & & & \multicolumn{2}{|c|}{ Sexo Masculino } & 00933 \\
\hline
\end{tabular}

Classe 1: Motivações sociais para a realização da cirurgia

A classe predominante nas falas dos entrevistados foram as motivações sociais, agregando $43,9 \%$ dos segmentos de texto. O conteúdo desta classe esteve centrado em situações sociais impossibilitadas por causa do peso e a preocupação com a imagem social. $O$ vocabulário evocado girou em torno dos seguintes termos: 'pessoa', 'magro', 'gordo', 'mudar', 'preconceito', e 'importante'. O peso, e suas comorbidades além do sofrimento causado pelo preconceito, parecem ter sido as principais motivações que levaram o obeso a procurar pelo procedimento cirúrgico. Como discurso simbólico desta classe, seria possível construir a seguinte sentença: "Para a pessoa que é gorda, mudar é importante, pois o magro não sofre preconceito".
Em estudo, Moliner e Rabuske (2008) citam as dificuldades relativas ao corpo como mediador das relações sociais e 0 preconceito sofrido pelo obeso, como disparadores à procura da cirurgia. Marcelino e Patrício (2011) também evidenciam os sentimentos de baixa autoestima e autodepreciação que estão relacionados com a ênfase midiática à inadequação do obeso aos padrões de beleza estabelecidos pela sociedade atual, acrescida das representações acerca das pessoas obesas. Em geral eles são vistos como preguiçosos, desleixados (Vilhena, Novaes, \& Rocha, 2008; Almeida et al., 2011; Goldenberg \& Ramos, 2007), sem caráter, e poucos acreditam em suas competências cognitivas, condições físicas, sociais e psicológicas (Almeida et al., 2011). Isso pode ser visto na seguinte expressão de um dos participantes: "As minhas 
motivações para fazer a cirurgia é como eu disse, que eu quero ser mais respeitável, mais aceitável, a sociedade te induz a fazer. Porque por mais que você não faça fica aquele preconceito. É como se você fosse ou indecente ou alguma coisa assim". [Participante, no 13 (P13)].

Analisando as variáveis de contraste podemos perceber que esse discurso aparece mais nas falas dos participantes do grupo pré-operatório $(p=0,00029)$ e do sexo feminino $(p<0,0001)$. Nas entrevistas foi citado vergonha de ir à praia, à academia, não poder viajar, e questões de mobilidade. Castro, Carvalho, Ferreira e Ferreira (2010) fazem uma relação entre função e qualidade de vida, quanto maior a redução de peso maior a funcionalidade do corpo e qualidade de vida. Portanto, o que 0 grupo pré espera da cirurgia é a possibilidade de mudança de vida, de conquistar a qualidade e funcionalidade que o peso não possibilitava ter. A pessoa obesa por vezes passa por situações vexatórias e de constrangimento, pois a acessibilidade é restrita pra quem foge do padrão estereotipado e é tido como não normal. Quanto ao grupo pós, entende-se que as pessoas que já fizeram a cirurgia ou já alcançaram, ou estão no caminho de emagrecer, estando, também, fascinados com a possibilidade de fazer coisas que antes não conseguiam por causa do peso e do isolamento social decorrente deste.

O imperativo da beleza magra é muito mais associado às mulheres do que aos homens (Nascimento, Bezerra, \& Angelim, 2013) e o maior número de mulheres entrevistadas não é por acaso. Esse grupo se sente mais pressionado socialmente a fazer a cirurgia para entrar nos padrões estéticos vigentes (Almeida et al., 2011), procurando mais pela redução de estômago, pois não se adéquam a nova moral da "boa forma" ou "onda fitness" (Goldenberg \& Ramos, 2007). Quanto aos motivos que as levam a essa busca cirúrgica, a imagem social - um fator externo ao indivíduo - pesa notavelmente para esse público, ficando fatores intrínsecos aos indivíduos, como por exemplo, prejuízos à saúde, em segundo plano.

Até entre os distúrbios alimentares a obesidade é o mais discriminado. As pessoas no geral são mais complacentes com quem sofre de bulimia e anorexia, pois essas patologias se aproximam mais da imagem "perfeita" do magro, padrão estético atual. Vilhena et al. (2008) dizem que é como se obeso fosse inteiramente responsável pela sua condição de doente e o anoréxico ou bulímico não. É como se estar abaixo do peso seja obrigatório no cenário social atual.

De todo o exposto, mostra-se relevante trazer o papel do psicólogo, cujo termo surgiu associado também na classe 1 (freq. $\left.75,00 \% ; X^{2}=11,58\right)$. Em virtude disso, procedeu-se uma análise de similitude em relação aos termos que apresentaram elevada coocorrência com a palavra 'psicólogo' no corpus (Figura 2).

Percebemos que as palavras mais associadas à 'psicólogo' foram 'importância', 'importante', 'pessoa', 'achar', 'mudar', 'gente', 'vida' o que confirma ainda mais o desejo de transformação e o psicólogo como auxiliador nesse processo tendo em vista as mudanças advindas com a cirurgia, não só na imagem corporal, como também no fator comportamental. Essa idéia pode ser resumida na seguinte sentença simbólica: "Acho o psicólogo importante (tem sua importância), pois a gente (ou pessoa) irá mudar de vida".

O papel do psicólogo para os participantes é de orientar, explicar e preparar o obeso para as transformações na vida após a cirurgia. Porém, a maioria deles só realiza 0 acompanhamento antes do procedimento por estar vinculado obrigatoriamente ao programa e a necessidade do laudo para a intervenção. 
Pelas entrevistas no pós-operatório a evasão do acompanhamento psicológico se deve a não cobertura dos planos de saúde e pela conquista de emagrecer. A exemplo disso: "Eu não fiz no pós, não precisei. Mas acho que para muitas pessoas é importante. Porque tem muita gente que se opera, mas tem mente de gordo, então não adianta nada. Vai perder peso por um tempo, mas depois pode engordar tudo. Antes e depois é importante. Antes é um preparo que você vai ter, de tudo que você vai passar. Outra coisa é você passar" (P6).

\section{FIGURA 2.}

Análise de similitude da palavra 'psicólogo'.

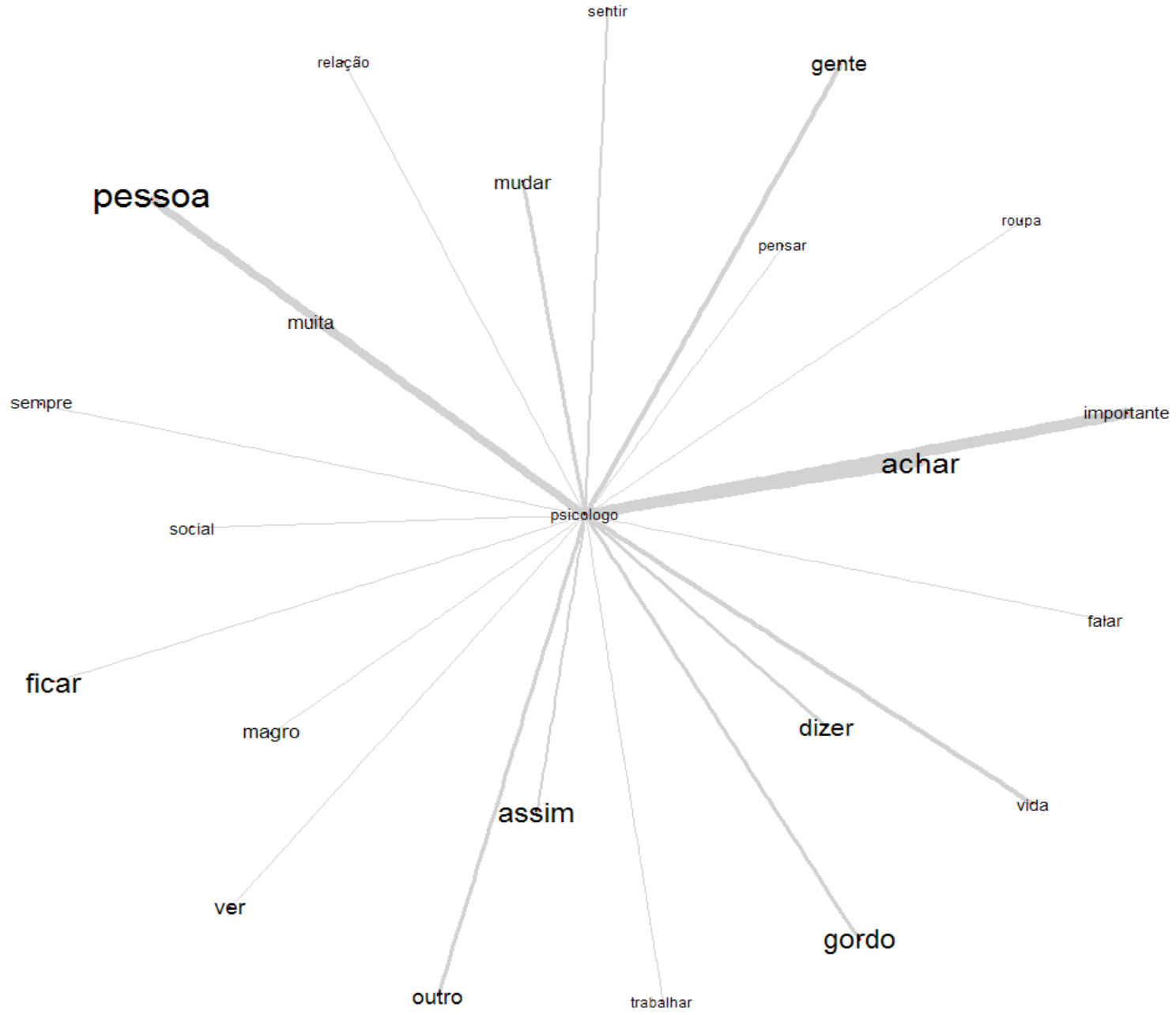

A cirurgia bariátrica, atualmente, tem em seus protocolos a previsão de avaliação e acompanhamento psicológico, desde a indicação da cirurgia, até os programas de reabilitação e reintegração sócio-familiar (Sebastiani \& Maia, 2005). Nesse processo é importante considerar o significado da alimentação e da obesidade para a família e para a pessoa, seus recursos para manejar limites e sentimentos de desconforto e frustrações. Também devem ser abordados os fatores relacionados à decisão do paciente em se submeter à cirurgia bariátrica e as suas expectativas com relação ao pós-operatório (Moliner \& Rabuske, 2008). 
Pelos estudos já realizados, não são poucas as possíveis consequências psicológicas após a cirurgia, ocorrendo situações danosas, tais como compulsão (Farias, Furtado, Morales, Santos, \& Coutinho, 2009; Machado et al., 2008), depressão (Porcu, Franzin, Abreu, Previdelli, \& Astolfi, 2011) e até mesmo suicídio (Tindle et al., 2010). Portanto, ressalta-se a importância da continuidade do psicólogo no processo, pois a alteração física resultante da cirurgia pode vir acarretar mudanças também psicológicas, que caso não sejam acompanhadas, favorece 0 aparecimento de complicações (Waidergorn, Lopes, \& Evangelista, 1999), pois a cirurgia em si é apenas o começo de um tratamento contínuo.

Constatou-se, enfim, que a expectativa dos obesos que fazem a cirurgia de redução de estômago, principalmente as mulheres, é que a cirurgia proporcione mudanças significativas de vida e resolver problemas de ordem psicossociais. Por isso também é tão importante a figura do psicólogo, pois o procedimento não é garantia de resolução de conflitos internos.

\section{Classe 2: Questões clínicas da cirurgia}

Com 29,2\%, nessa classe foram associadas os termos: 'cirurgia', 'mês', 'saúde', 'médico', 'dieta', 'perder', 'expectativa', 'líquido', 'peso', 'exame', 'começar', 'minha', 'tentar', 'motivo', 'plano'. O uso desses termos revela a preocupação com 0 processo cirúrgico em si, as implicações na saúde e as transformações, principalmente, no primeiro mês. A sentença simbólica que poderia expressar a idéia da classe é entendida como: "Procurei o médico pra tentar fazer a cirurgia por motivos de saúde. Minha expectativa era perder peso no primeiro mês com a dieta líquida".

A obesidade mórbida é acompanhada de uma série de complicações que comprometem a saúde de maneira geral e põe em risco a vida do indivíduo. As principais doenças associadas a obesidade são doenças cardíacas, diabetes, hipertensão arterial, dislipidemias, acidente vascular cerebral, aterosclerose e tipos específicos de câncer além de aumento da mortalidade global e uma diminuição na vida útil de 10 anos (Kubic, Gill, Laffin, \& Karmali, 2013). Conforme a gravidade da obesidade aumenta, aumenta também as complicações médicas e 0 risco de mortalidade. Por esse motivo ainda percebe-se também que o foco da cirurgia está muito mais na figura do médico do que em um trabalho multidisciplinar visto que outros profissionais não foram aludidos. Marchiolli et al. (2005) falam da importância do cuidado multidisciplinar, justamente pelo motivo da obesidade também carregar, além das complicações clínicas, aspectos sociais e psíquicos.

Analisando o contraste dessa classe, foi percebida uma maior significância no sexo masculino ( $p=0,00933)$. Vê-se, então, que as consequências da obesidade para a saúde foram mais mencionadas de forma recorrente pelos participantes homens. A cirurgia para esse grupo parece ser considerada a forma de minimizar problemas de saúde que já enfrentam, diminuir 0 risco de ocorrência de comorbidades e aumentar a longevidade (Moliner \& Rabuske, 2008), diferentemente das mulheres, cujo contraste apareceu na Classe 1 relacionado a mudanças na imagem social. Ao que parece, os participantes do sexo masculino não identificaram tanto os aspectos psicossociais da obesidade se focando apenas nas questões clínicas. Van Hout, Van Oudheusden, Kasuska e Van Heck (2006b) constataram diferenças entre mulheres e homens obesos envolvidos no processo da bariátrica. As primeiras são mais vulneráveis ao desenvolvimento de psicopatologias no pré-operatório, comendo muito mais de forma emocional, além do que são mais insatisfeitas com o corpo. Participantes do sexo masculino tiveram médias significativamente menores no 
funcionamento físico, tendendo a ter menores escores na dor e na percepção geral de saúde.

Resumindo, essa classe expôs a preocupação com a saúde, tendo na figura do médico 0 ator principal da equipe, e, também a preocupação com o primeiro mês da cirurgia. Esses fatores foram mais citados pelos participantes do sexo masculino, o que aliada à classe anterior, mostra diferenças no sentido da cirurgia para homens e mulheres.

Classe 3: Mudanças na alimentação associadas à cirurgia

A classe mudanças na alimentação representou $26,9 \%$ dos segmentos de texto. Ela trata das mudanças na alimentação e na dinâmica fisiológica da pessoa submetida à redução de estômago. As formas mais aludidas foram: 'comer'; 'doce'; 'dumping'; 'comida'; 'passar'; 'beber'; 'só'; 'copo'; 'lembrar'; 'carne'.

De acordo com Marcelino e Patrício (2011) o tipo e a quantidade da alimentação orientada para o pós-operatório variam de acordo a população e o método cirúrgico adotado. Por outro lado há necessidade em todos os procedimentos de cuidados no processo da readaptação alimentar, pois é nesta fase que o operado sofre com os grandes sacrifícios, o que exige força de vontade, autocontrole e determinação. De acordo com o autor, os sujeitos possuem práticas alimentares inapropriadas, na quantidade e na qualidade dos alimentos e na dinâmica cotidiana de se alimentar. A fala a seguir exemplifica a consciência de que os hábitos alimentares excessivos não serão mais possíveis: "Acho que vai ter algum tipo de alimento que não vou poder comer, por diminuir a quantidade. Quanto a isso estou bem consciente. Agora acho que tem alguns alimentos que não vou comer. Não posso comer" (P01).

Importante destacar que a alimentação continua sendo um elemento central no discurso. Pessoas obesas estabelecem padrão de reação aos conflitos baseado na alimentação, relacionado a dificuldades em organizar emoções. Se triste, come. Se feliz, come. No pós-operatório, como a alimentação excessiva não é possível, os obesos operados então podem passar a comer mais, em intervalos curtos, alimentos de fácil ingestão como doces e petiscos (Machado et al., 2008).

Podemos perceber que uma das mudanças esperadas e observadas são as rejeições de alguns tipos de alimentos, como doce, carne, entre outros. Isso também foi observado por Moliner e Rabuske (2008), ao também constatarem que os participantes esperam que 0 estômago imponha limites no comportamento alimentar diante da quantidade e tipo dos alimentos. A exemplo dessa noção: "Não importava a velocidade ou o tamanho, eu sufocava mesmo quando comia carne vermelha. Foi uma coisa que acabei diminuindo bastante, até hoje eu como bem menos carne em porcentagem, se for comparar ao que comia antigamente, claro que será sempre menos. Mas em porcentagem eu como muito mais carne branca e peixes do que vermelha, porque ficou essa idéia mesmo de sufocamento" (P04).

O tratamento cirúrgico da obesidade não é uma alternativa à dieta, mas um método para aplicá-la. O comportamento alimentar no pós-operatório é um mediador importante da perda de peso, e, por conseguinte, influencia nos resultados e sucesso da cirurgia. De acordo com Van Hout et al. (2006a) as mudanças e o cumprimento de aconselhamento dietético são necessários, especialmente em relação à quantidade $\mathrm{e} a$ velocidade da ingestão de alimentos. Essas mudanças alimentares não são completamente facilitadas, ou forçadas, pelas mudanças internas que resultam da operação, os pacientes também têm papel ativo no processo em ajustar seu comportamento alimentar. 
A síndrome de dumping também foi citada nesta classe. Zagury, Moreira, Guedes, Coutinho e Appolinario (2004) definem a síndrome de dumping como uma reação resultante da ingestão de alimentos calóricos seguido de rápido esvaziamento para o intestino delgado. Segundo o autor os efeitos mais frequentes são taquicardia, palpitações, sudorese, e náusea. Com a ocorrência em praticamente todas as operações gástricas (Loss, Souza, Pitombo, Milcente, \& Madureira, 2009), a síndrome não é vista pelos participantes como um contra da cirurgia por ser uma sensação passageira e que pode ser evitada.

Apesar do mal-estar que causa, 0 dumping pode ser tido como algo positivo, pois sua ocorrência ajuda a limitar a quantidade de alimentos ingeridos. Os pacientes com a síndrome devem aprender a evitar 0 dumping por alterações na quantidade e qualidade do que é comido (Zagury et al., 2004). Por outro lado a insegurança em relação ao tipo de alimento pode suscitar medo de se alimentar, o que pode evoluir para distúrbios nutricionais (Loss et al., 2009).

Quando analisados os contrastes dessa classe temos maior significância de participantes do grupo pós-operatório $(p=$ 0,00259 ), os quais já passaram e ainda passam por essas mudanças no biológico e na alimentação. No grupo pré-operatório, mesmo sendo esclarecidos dos fatos e do que pode acontecer, inclusive o dumping, não passaram ainda na pele 0 efeito dessa síndrome, e ficam na esperança de que com a cirurgia acabem todos os problemas e, para isso, vale passar pelo sacrifício (Magdaleno et al., 2009; Marchiolli et al., 2005). Em suma, percebemos que os participantes são conscientes das restrições em quantidade e tipo de alimentos ingeridos após a cirurgia, e da imposição do estômago recusando excessos por meio do dumping, o que não foi tido como contra ou aspecto negativo do processo.

\section{Análise Geral das Classes}

Analisando-se em conjunto, no geral podemos concluir pela divisão da CHD que as classes que mais se aproximam são as classes 1, Motivações Sociais, e a classe 3, Mudanças na Alimentação. A transformação sobrevinda da cirurgia, através da dieta e restrições impostas pelo tamanho reduzido do estômago, convém para ficar bonito, apresentável e aceitável, e não para ficar saudável, visto a distância com a classe 2 , Questões Clínicas e de Saúde. O processo cirúrgico pode estar sendo vendido como uma solução rápida de perda de peso e ganho de um corpo estético perfeito.

Aponta-se também que a cirurgia tem sentidos diferentes entre homens $e$ mulheres, os quais os primeiros se preocupam mais com questões da saúde e comorbidades aliadas a obesidade e as segundas, sendo mais pressionadas socialmente para ter um corpo perfeito, se inquietam mais com a imagem social e as representações atreladas ao ser obeso. 0 grupo pré-operatório, apesar de cientes das transformações e limitações do estômago reduzido, acha que vale a pena o sacrifício para se livrar do sofrimento social e físico que o peso carrega, cenário que se inverte após a realização do procedimento. 0 grupo pós-operatório já não fala de preconceito e dificuldades sociais, sugerindo uma melhoria no social pós-operatório. Porém 0 discurso sobre as modificações na alimentação e no fisiológico é uma constante.

0 presente estudo comporta algumas limitações. $O$ grupo pós foi composto apenas por pessoas que haviam realizado 0 procedimento por via de convênios com planos particulares de saúde. As cirurgias de redução de estomago pelo SUS no estado de Sergipe estavam suspensas no período da coleta, fato que impossibilitou 0 contato com pessoas que realizaram a cirurgia por esse meio. Sugere-se em 
pesquisas posteriores fazer uma comparação entre esses dois grupos, pessoas que fizeram a cirurgia pelo SUS e pessoas que fizeram a cirurgia por convênios e planos particulares, analisando diferenças e analogias. Outro fato digno de nota, pela dificuldade de contatos com 0 público estudado, procurou-se espaços onde esses se encontravam, hospitais e grupos multidisciplinares, 0 que pode ter homogeneizado a amostra. Sugere-se em trabalhos subsequentes realizar a coleta fora desses espaços. Outro aspecto é a pequena proporção de participantes do sexo masculino, justificada pela maior procura do público feminino ao procedimento, o que poderia resultar em observações diferentes.

Este estudo contribui para 0 conhecimento dos pacientes pré e pósoperatório de cirurgia bariátrica no que diz respeito as diferenças entre os discursos de tais grupos. Os dados apontam que dentre os fatores envolvidos na tomada de decisão para o procedimento cirúrgico, apesar de terem sido citados os motivos de saúde, o que aparentemente pesa para o grupo pesquisado são as imposições da sociedade por um corpo ideal $e$ transformado, sendo as mulheres as mais cobradas nesse sentido o que justifica a maior procura pela cirurgia. A diferença entre os sexos é pouco abordada em estudos com essa temática, sendo este trabalho um acréscimo no entendimento de pacientes bariátricos.

Enfim, o profissional psicólogo ainda é negligenciado e, apesar de ser considerado importante no processo, não há continuidade no tratamento psicológico. Isso evidencia uma contradição, pois apesar dos motivos que levam à cirurgia serem predominantemente psicossociais, esse fator não é levado em conta na prática, sendo valorizadas apenas as implicações clínicas.

\section{REFERÊNCIAS}

Almeida, G. A. N., Giampietro, H. B., Belarmino, L. B., Moretti, L. A., Marchini, J. S., \& Ceneviva, R. (2011). Aspectos psicossociais em cirurgia bariátrica: A associação entre variáveis emocionais, trabalho, relacionamentos e peso corporal. $A B C D$ : Arquivos Brasileiros de Cirurgia Digestiva, 24, 226-231. doi: 10.1590/S0102-67202011000300009

Almeida, R. A., \& Malagris, L. E. N. (2011). A prática da psicologia da saúde. Revista da Sociedade Brasileira de Psicologia Hospitalar, 14, 183-202.

Brasil (2011). Ministério da Saúde. Prevalência de excesso de peso em adultos. Acesso em 08 de setembro de $2013 . \quad$ Disponível em:http://tabnet.datasus.gov.br/cgi/idb2 011/g07_08.htm

Brasil (2013). Ministério da Saúde. Portaria $n^{\circ}$ 425, de 19 de março 2013.

Camargo, B. V., \& Justo, A. M. (2013). IRAMUTEQ: Um Software Gratuito para Análise de Dados Textuais. Temas em Psicologia, 21, 513-518. doi: 10.9788/TP2013.2-16.

Castro M. R., Carvalho R. S., Ferreira V. N., \& Ferreira M. E. C. (2010). Função e Imagem Corporal: Uma análise a partir do discurso de mulheres submetidas à cirurgia bariátrica. Revista Brasileira de Ciências do Esporte, 32, 167-183. doi: 10.1590/S0101-32892010000200012.

Engström, M., Wiklund, M., Ólsen, M. F., Lönroth H., \& Forsberg, A. (2011). The meaning of awaiting bariatric surgery due to morbid obesity. The Open Nursing Journal, 5, 1-8. doi: 10.2174/1874434601105010001.

Farias P. M., Furtado C. A. S., Morales G., Santos L. C., \& Coutinho V. (2009). Compulsão alimentar em pacientes submetidos a cirurgia bariátrica. Revista Brasileira de Nutrição Clínica, 24 , 137-42. doi: 10.1590/S010267202008000400007. 
Gabarra, L. M., \& Crepaldi, M. A. (2009). Aspectos psicológicos da cirurgia de amputação. Aletheia, 30, 59-72.

Goldenberg M., \& Ramos M. S. (2007). A civilização das formas: 0 corpo como valor. In: Goldenberg. M. (Org.). Nu e Vestido: Dez antropólogos revelam a cultura do corpo carioca (pp.19-40). Rio de Janeiro: Record.

Kubic, J. F., Gill R. S., Laffin M., \& Karmali S. (2013). The impact of bariatric surgery on psychological health. Journal of Obesity. doi:10.1155/2013/837989

Loss, A. B., Souza, A. A. P., Pitombo, C. A., Milcent, M., \& Madureira, F. A. V. (2009). Avaliação da síndrome de dumping em pacientes obesos mórbidos submetidos à operação de bypass gástrico com reconstrução em $Y$ de Roux. Revista do Colégio Brasileiro de Cirurgiões, 36, 413-419. doi: $10.1590 / S 0100-$ 69912009000500009.

Machado, C. E., Zilberstein, B., Cecconello, I., \& Monteiro, M. (2008). Compulsão alimentar antes e após a cirurgia bariátrica. $A B C D$ : Arquivos Brasileiros de Cirurgia Digestiva, 21, 185-191. doi: 10.1590/S0102-67202008000400007.

Magdaleno, R., Jr. (2009). Vivências emocionais de mulheres submetidas à cirurgia bariátrica no Hospital das Clínicas da Unicamp: Um estudo clínico-qualitativo (Tese Doutorado). Universidade Estadual de Campinas, Faculdade de Ciências Médicas, Campinas.

Marcelino L. F., \& Patrício Z. M. (2011). A complexidade da obesidade e 0 processo de viver após a cirurgia bariátrica: Uma questão de saúde coletiva. Ciência \& Saúde Coletiva, 16, 4767-4776. doi: 10.1590/S141381232011001300025.

Marchiolli, A. C. D., Marchiolli, P. T. O., \& Silva, L. B. C. (2005). As consequências psicossociais da cirurgia de redução de estômago. Psicologia da Saúde, 13, 175-214.
Moliner, J., \& Rabuske M. M. (2008). Fatores biopsicossociais envolvidos na decisão de realização da cirurgia bariátrica. Psicologia: Teoria e Prática, 10, 44-60.

Nascimento C. A. D., Bezerra S. M. M. S., \& Angelim E. M. S. (2013). Vivência da obesidade e do emagrecimento em mulheres submetidas à cirurgia bariátrica. Estudos de Psicologia, 18, 193-201. doi: 10.1590/S1413294X2013000200004.

Porcu, M., Franzin R., Abreu P. B., Previdelli I. T. S., \& Astolfi M. (2001). Prevalência de transtornos depressivos e de ansiedade em pacientes obesos submetidos à cirurgia bariátrica. Acta Scientiarum Health Sciences, 33, 165171.

doi: 10.4025/actascihealthsci.v33i2.7653.

Sebastiani, R. W., \& Maia, E. M. C. (2005). Contribuições da psicologia da saúdehospitalar na atenção ao paciente cirúrgico. Acta Cirúrgica Brasileira, 10, 50-55. doi: 10.1590/S010286502005000700010.

Segal, A., \& Fandiño, J. (2002). Indicações e contra-indicações para realização das operações bariátricas. Revista Brasileira de Psiquiatria, 24, 68-72. doi: 10.1590/S1516-44462002000700015.

Silva, S. S. P., \& Maia, A. C. (2013). Patients' perceptions, health and psychological changes with obesity treatment: Success and failure in a triangulation study. Health, 5, 17501759 .

doi: 10.4236/health.2013.511236.

Tindle, H. A., Omalu B., Courcoulas, A., Marcus, M., Hammers, J., \& Kuller, L. H. (2010). Risk of suicide after longterm follow-up from bariatric surgery. The American Journal of Medicine, 123, 1036-1042. doi: 10.1016/j.amjmed.2010.06.016.

Van Hout, G. C. M., Boekestein, P., Fortuin, F. A. M., Pelle, A. J. M., \& Van Heck G. L. (2006a). Psychosocial functioning following bariatric surgery. Obesity 
Surgery, 16, 787- 794 . doi: 10.1381/096089206777346808.

Van Hout G. C. M., Van Oudheusden I., Kasuska A. T., \& Van Heck G. L. (2006b). Psychological profile of candidates for vertical banded gastroplasty. Obesity Surgery, 16, 6774. doi: 0.1381/096089206775222023.

Vilhena J., Novaes J. V., \& Rocha L. (2008) Comendo, comendo e não se satisfazendo: Apenas uma questão cirúrgica? Obesidade mórbida e o culto ao corpo na sociedade contemporânea. Revista Mal-estar e Subjetividade, 8, 379-406.

Waidergorn, L., Lopes, C., \& Evangelista, R. (1999). $O$ acompanhamento psicológico do obeso mórbido. Psikhê, 4, 74-82.

Zagury, L., Moreira R. O., Guedes E. P., Coutinho W. F., \& Appolinario J. C. (2004). Insulinoma misdiagnosed as dumping syndrome after bariatric surgery. Obesity Surgery, 14, 120-123. doi: 10.1381/096089204772787419. 